\title{
Decreased expression of extracellular matrix proteins and trophic factors in the amygdala complex of depressed mice after chronic immobilization stress
}

\author{
Soonwoong Jung, Younghyurk Lee, Gyeongwha Kim, Hyeonwi Son, Dong Hoon Lee, Gu Seob Roh, \\ Sang Soo Kang, Gyeong Jae Cho, Wan Sung Choi and Hyun Joon Kim
}

\begin{abstract}
Background: The amygdala plays an essential role in controlling emotional behaviors and has numerous connections to other brain regions. The functional role of the amygdala has been highlighted by various studies of stress-induced behavioral changes. Here we investigated gene expression changes in the amygdala in the chronic immobilization stress (CIS)-induced depression model.

Results: Eight genes were decreased in the amygdala of CIS mice, including genes for neurotrophic factors and extracellular matrix proteins. Among these, osteoglycin, fibromodulin, insulin-like growth factor 2 (Igf2), and insulin-like growth factor binding protein 2 (lgfbp2) were further analyzed for histological expression changes. The expression of osteoglycin and fibromodulin simultaneously decreased in the medial, basolateral, and central amygdala regions. However, Igf2 and Igfbp2 decreased specifically in the central nucleus of the amygdala. Interestingly, this decrease was found only in the amygdala of mice showing higher immobility, but not in mice displaying lower immobility, although the CIS regimen was the same for both groups.
\end{abstract}

Conclusions: These results suggest that the responsiveness of the amygdala may play a role in the sensitivity of CIS-induced behavioral changes in mice.

\section{Background}

Stress is believed to cause various diseases, yet has also been shown to have some beneficial effects [1]. Stress can lead to somatic abnormalities and can evoke psychiatric illnesses such as anxiety disorders and major depressive disorder [2]. Therefore, to elucidate a pathway to recovery, much research has focused on investigating why various stresses result in such diseases [3].

Recent clinical and pre-clinical studies have revealed that psychiatric illnesses such as depression and anxiety are due to over-activated and/or depressed functions of specific brain regions. Particular emphasis has been placed on examining the prefrontal cortex, hippocampus, and amygdala due to the different structure and

\footnotetext{
*Correspondence: kimhj@gnu.kr

Department of Anatomy and Neurobiology, Institute of Health Sciences, Medical Research Center for Neural Dysfunction School of Medice, Gyeongsang National University, 92 Chilam-dong, Jinju, 660-751, South Korea
}

function of these regions in psychiatric cases compared with normal controls [4,5]. Notably, the importance of the amygdala in this pathway has been emphasized because of its many neural connections to other brain regions [6].

The amygdala was named for its almond-like shape and is known for its role in the formation and recall of emotional memories [7]. Because the amygdala consists of four nuclei (lateral, medial, central, and basal nucleus), it is also often referred to as the amygdala complex. Each nucleus has its own neural circuit that is distinct from the other nuclei in the amygdala complex and other brain areas. Through these neural circuits, the amygdala plays an important role in integrating and responding to external sensory information [6].

Determining the changes in the brain that occur with different mental illnesses will provide invaluable clues for understanding and formulating an earlier prognosis 
and developing proper treatments for anxiety disorders and depression [8]. These changes may be epigenetically evoked by environmental changes such as stress. Therefore, much work has been performed in various laboratories worldwide focusing on the elucidation of gene expression patterns and changes in experimental animal models $[5,9]$. Our laboratory previously established a chronic immobilization stress (CIS)-induced anxiety and depression mouse model, and changes in gene expression were reported in the prefrontal cortex [10]. In our current study, we found that a few extracellular matrix $(E C M)$ proteins and trophic factors were reduced in the amygdala of CIS-induced depressed mice compared with control mice.

\section{Methods}

\section{Analysis of amygdala gene expression in anxious and depressed mice}

To analyze the gene expression pattern in the amygdala of anxious and depressed mice, we performed cDNA microarray analysis on micro-punched amygdala tissue from ICR mice experiencing CIS [10]. Briefly, 7-weekold male ICR mice were subjected to a 15-day ( $2 \mathrm{~h} /$ day) CIS paradigm, and CIS-induced behavioral changes were measured in an open field test, an elevated plus maze, and a forced swim test (FST). For microarray analysis, total RNA from the amygdala of control (CTL) and stressed (STR) mice was obtained using a micro-punch (diameter $1.21 \mathrm{~mm}$, Stoelting, IL, USA) and pooled into one sample consisting of 10 mice from each group. A DNA microarray assay was done once at the DNA Microarray Core Facility (University of Kentucky, Lexington, KY) using Affymetrix equipment, protocols, and the GeneChip ${ }^{\circledR}$ Mouse Expression Set 430 2.0. CISresponsive genes were selected if their expression values, which were signal intensities of probe hybridization provided by Affymetrix microarray assay equipment [11], changed by 2 -fold or greater.

\section{Confirmation of changes in gene expression in the amygdala \\ Animals and treatments}

To confirm gene expression changes, male C57BL/6 mice were used. Male 7-week-old C57BL/6 mice (Samtako, Co. Ltd., Korea) were habituated for 1 week before experimentation in a specific pathogen-free-grade animal facility at Gyeongsang National University School of Medicine. Temperature and humidity were $22{ }^{\circ} \mathrm{C}$ and $50-60 \%$, respectively, and mice were reared under a 12 h light/dark cycle (lights on at 6 a.m.). Mice were supplied with free access to laboratory chow and water. CIS was carried out as previously described [10]. Briefly, mice were repeatedly placed in a restrainer for $2 \mathrm{~h} /$ day for 15 days under 200 lux light conditions. Body weight and food intake were evaluated every other day. All procedures were performed in accordance with an approved protocol (GLA-100917-M0093) by Gyeongsang National University Institution Animal Care \& Use Committee (GNU IACUC).

\section{Behavioral assessments}

To measure the depression level of CIS mice, a sucrose preference test (SPT) and FST were conducted as depicted in Figure 1A. The SPT was performed with some modifications as previously described [12] to determine symptoms of anhedonia. Briefly, mice were habituated for $48 \mathrm{~h}$ with $0.1 \mathrm{M}$ sucrose solution. Following a 12-h water deprivation period, consumption of sucrose solution and water was determined for $6 \mathrm{~h}$. The sucrose preference was represented as the ratio of sucrose-to-water consumption. The FST method was performed as previously described [10]. Mice were subjected to pre-swimming for $5 \mathrm{~min}$ on the day prior to experimentation. Mobility was defined as a change in $5 \%$ of the recorded pixels (EthoVision, Noldus Information Technology, The Netherlands). The water in the chamber was changed between each mouse.

\section{Quantitative PCR analysis}

Mice were anesthetized with $\mathrm{CO}_{2}(\mathrm{CTL} ; \mathrm{n}=9$, STR; $\mathrm{n}=9$ ) and decapitated. Brains were removed and quickly frozen in isopentane pre-chilled in liquid nitrogen. Amygdaloid tissue was obtained using a micro-punch $0.8 \mathrm{~mm}$ deep from the caudal end forward to $-1.58 \mathrm{~mm}$ from the bregma [13]. Tissue punch samples were stored at $-70{ }^{\circ} \mathrm{C}$ until use. Total RNA was extracted using Trizol reagent and further purified using an RNeasy kit (Qiagen Inc., Valencia, CA, USA). To verify the microarray results, quantitative PCR (Q-PCR) was performed with gene-specific primers (Table 1). First strand cDNA was synthesized from $1 \mu \mathrm{g}$ total RNA with oligo $(\mathrm{dT})_{15}$ primers. Q-PCR amplification and relative quantification were achieved using a LightCycler 480 (Roche) as previously described [14]. The data are presented as mRNA expression levels and were normalized to GAPDH expression as an internal control.

\section{Immunohistochemistry}

Immunohistochemistry was performed as previously described [19], with several modifications. Briefly, fixed brains were sectioned at $30-\mu \mathrm{m}$ thickness and incubated with anti-fibromodulin (Santa Cruz Biotechnology, Cat \#; L2807, 1:100), osteoglycin (Santa Cruz Biotechnology, Cat \#; A0108, 1:100), insulin-like growth factor 2 (Igf2; Abcam, Cat \#; ab63984, 1:200), or insulin-like growth factor binding protein 2 (Igfbp2; R\&D Systems, Cat \#; AF797, 1:100) at $4{ }^{\circ} \mathrm{C}$ overnight. Signals were visualized using Alexa Flour-conjugated secondary antibodies, and 
(A)

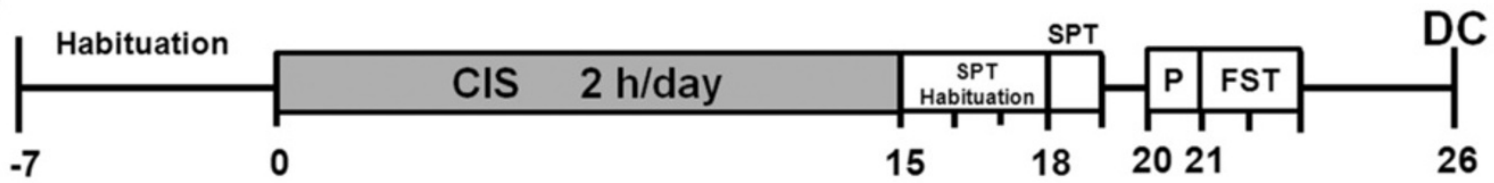

(B)

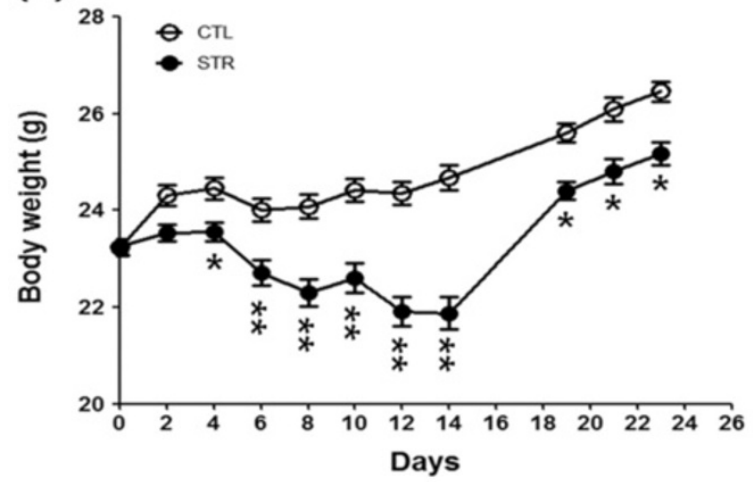

(D)

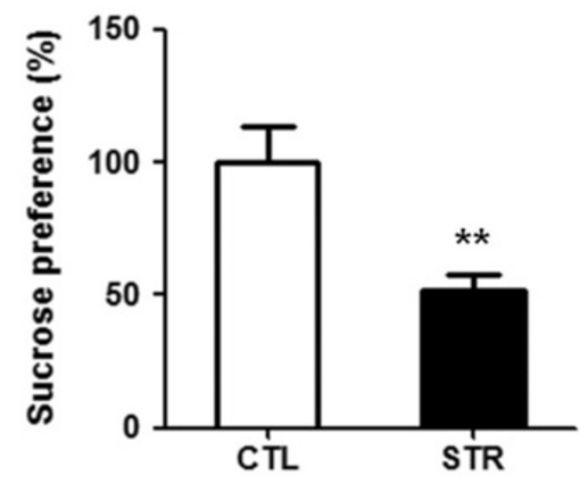

(C)

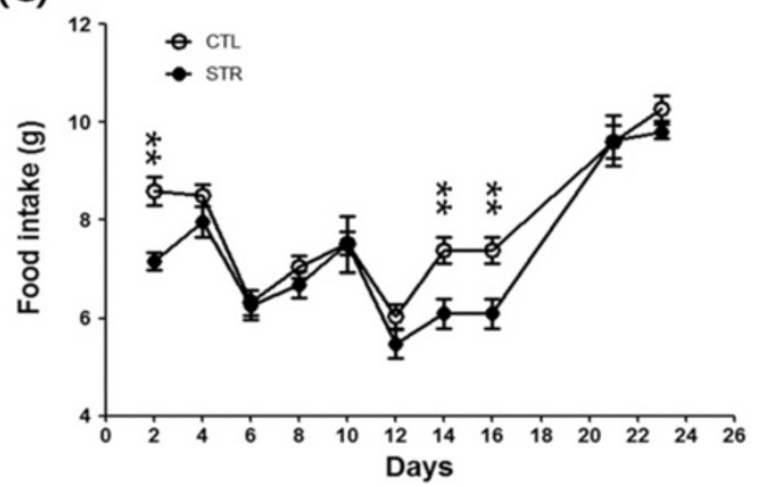

(E)

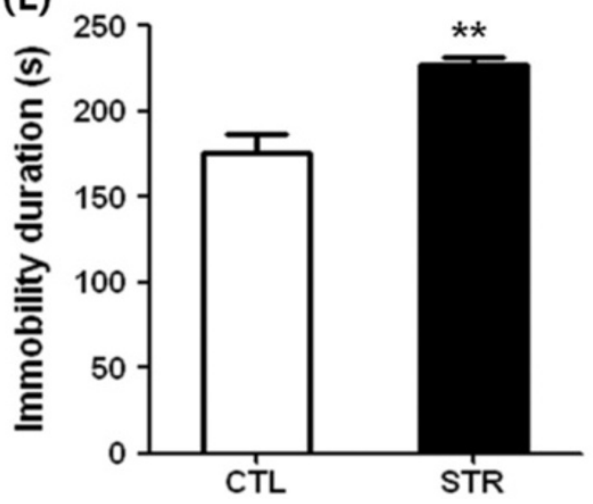

Figure 1 A schematic CIS paradigm and the result of behavioral assessments. (A) A schematic drawing of CIS (2 h per day for 15 days) and behavioral assessments: sucrose preference test (SPT) and forced swim test (FST). After behavioral assessments, the experimental animals were killed by decapitation (DC) on day 26 for Q-PCR analysis. On the day before FST, mice were adapted to swimming with a pre-swimming (P) session. The body weight (B) and food intake (C) were measured every other day during experimentation except for during the SPT period. The significance of the mean difference between the stressed (STR, $n=10)$ and control $(C T L, n=10)$ groups was measured with a $t$-test on each day. The STR group showed body weight reduction over the 15-day CIS period. Significant mean differences in food intake were only found on days 2, 14, and 16 after the experiment began. Sucrose preference of the STR group was significantly decreased (D), and immobility duration of the STR group increased $(\mathbf{E})$ compared with CTL, indicating that STR mice were more depressed than CTL mice. Data are the means \pm SEM. *, $P<0.05 ;{ }^{* *}, P<0.01$ between $C T L$ and STR groups.

nuclei were stained with DAPI. Signal specificity was confirmed with a negative control; sections that were incubated with no primary antibody showed no specific signals. Digital images were captured and documented (Olympus, Tokyo, Japan). The signal densities were analyzed on similar sections from three different mice from each group with an image processing program $(\mathrm{NIH}$ Image J, Bethesda, MD).

\section{Statistics}

Data were evaluated using a one-way ANOVA and a post-hoc (Tukey's multiple-comparison test) test (GraphPad Prism, Ver 5.01). The $t$-test was used to compare two groups (SigmaStat Ver. 10.0). Statistical significance was set at $\mathrm{P}<0.05$.

\section{Results}

Previously, we used microarray analysis and reported cortical gene expression changes in anxious and depressed mice experiencing CIS [10]. We saved amygdala tissues from the mice in that study and performed DNA microarray analyses using them. Therefore, the microarray results presented in this study are from the amygdala of CIS-induced anxious and depressed mice.

To select genes for further study from the microarray data, we used two criteria. The first was a gene 
Table 1 Primers used for Q-PCR analysis

\begin{tabular}{|c|c|c|c|}
\hline \multirow{3}{*}{$\begin{array}{l}\text { Gene Name } \\
\text { Fibromodulin }\end{array}$} & \multicolumn{2}{|r|}{ Primer sequence $\left(5^{\prime}-3^{\prime}\right)$} & \multirow{2}{*}{$\begin{array}{l}\text { Reference or GenBanl } \\
\text { Accession number } \\
{[15]}\end{array}$} \\
\hline & Forward & GAG CCT CTG CTC ATC CTT TG & \\
\hline & Reverse & CTG GTT TGG CTT TTG TGG AT & \\
\hline \multirow[t]{2}{*}{ Osteoglycin } & Forward & TGA TGC TGT ACC ACC ATT GC & [16] \\
\hline & Reverse & ATT CCA GGT CGT TAT GGT CC & \\
\hline \multirow[t]{2}{*}{ Procollagen C-proteinase enhancer protein } & Forward & CTG AGC ACC AGT TTT GTG GG & NM 008788 \\
\hline & Reverse & CCA GCC TCT TTG AGT CGT CA & \\
\hline \multirow[t]{2}{*}{ Procollagen, type I, alpha 2} & Forward & CTG CTG GAG TCA AGG GTG AT & NM 007743 \\
\hline & Reverse & CTG TCT CCT TGC TTG CCA GT & \\
\hline \multirow[t]{2}{*}{ Thrombomodulin } & Forward & ATG CGT GGA GCA TGA GTG & [17] \\
\hline & Reverse & CTG GCA TCG AGG AAG GTC & \\
\hline \multirow[t]{2}{*}{ Growth differentiation factor 10} & Forward & GGT GGA CTT CGC AGA CAT CG & NM 173404 \\
\hline & Reverse & GAT GGT GGC ATG GTT GGA TG & \\
\hline \multirow[t]{2}{*}{ Insulin-like growth factor 2} & Forward & CGC TTC AGT TTG TCT GTT CGG & [18] \\
\hline & Reverse & TGG GTG GTA ACA CGA TCA GG & \\
\hline \multirow[t]{2}{*}{ Insulin-like growth factor binding protein 2} & Forward & GGC GCG GGT ACC TGT GAA AA & [18] \\
\hline & Reverse & TCT CCT GCT GCT CGT TGT AG & \\
\hline \multirow[t]{2}{*}{ Insulin-like growth factor binding protein 4} & Forward & CCA TCC AGG AAA GCC TGC AG & [18] \\
\hline & Reverse & TGG AAG TTG CCG TTG CGG TCA CAG & \\
\hline \multirow[t]{2}{*}{ Gapdh } & Forward & TGC CGC CTG GAG AAA CCTG C & [14] \\
\hline & Reverse & TGA GAG CAA TGC CAG CCC CA & \\
\hline
\end{tabular}

expression change of more than 2-fold, and the second was an expression value greater than 500 in either group of control or stressed animals. The expression value was hybridization signal intensity provided by the Affymetrix microarray system software. Consequently, only 10 probes (eight genes) satisfied the criteria (Table 2), a number that was very small compared to the large number of probes on the chip. Also, these genes were functionally categorized into only two groups, ECM and trophic factors. Fibromodulin, osteoglycin, thrombomodulin, procollagen $\mathrm{C}$-proteinase enhancer protein, and procollagen type 1, alpha 2 are ECM proteins; growth differentiation factor 10 (Gdf10), Igfbp2, and Igfbp4 belong to the trophic factor family. Because the microarray was done once without replicate, confirmation of gene expression changes should be done with further analyses.

To confirm the gene expression changes of selected genes using another experimental animals and Q-PCR analyses, we produced CIS-induced depressed mice using a similar yet slightly different paradigm (Figure 1A) from our previous study [10]. We wanted to focus only on the depression-like behaviors evoked by CIS. For this, two behavioral tests, SPT and FST, were conducted to assess the depression level of the mice after a 15-day CIS treatment. In addition, we replaced the ICR mouse strain with $\mathrm{C} 57 \mathrm{BL} / 6$, because the latter are more popularly used. During the CIS procedure, body weight and food intake were checked every other day (Figure 1B and C). We observed a significant reduction in body weight in the stressed group compared with controls, although there was no significant difference in their food intake during the mid-stage of CIS treatment. Therefore, the body weight change may have been due to differences in energy expenditure between the control and stressed groups. As expected, the stressed group showed a lower sucrose preference and higher immobility duration than the control group, implying increased depression in the stressed group (Figure 1D and E).

After confirmation of behavioral changes, Q-PCR was conducted to verify mRNA expression changes in the CIS-induced depressed animals. Since the list (Table 2) contained two genes of Igf-binding proteins, Igfbp 2 and $I g f b p 4$, we hypothesized that the expression of Igf-related genes could be influenced by CIS. We again analyzed the microarray data to see whether other Igf-related genes were changed. It was found that Igf2 expression was decreased by 1.7 -fold with high expression level (expression value of control group was 1449.4) and other Igf-related genes showed neither remarkable changes nor high expression level (data not shown). Therefore, we included Igf2 in Q-PCR analysis. Because the change in expression was less than 2-fold, the genes listed in Table 2 do not include Igf2. From Q-PCR analysis, however, Igf2 showed significantly lower expression in the STR group compared with the CTL, which was similar to other gene candidates (Figure. 2). However, mRNA 
Table 2 List of known genes that showed a 2-fold or greater change in expression in the amygdala of CISinduced depressed mice

\begin{tabular}{llll}
\hline GenBank & Gene Description & \multicolumn{2}{l}{ Expression Values } \\
\cline { 3 - 4 } & & Control & Stress \\
\hline NM_021355.1 & fibromodulin & $\mathbf{6 0 3}$ & $\mathbf{1 8 6}$ \\
BB532202 & fibromodulin & $\mathbf{1 2 7 5}$ & $\mathbf{5 2 3}$ \\
BC021939.1 & osteoglycin & $\mathbf{7 3 7}$ & $\mathbf{2 4 9}$ \\
BB542051 & osteoglycin & $\mathbf{7 0 5}$ & $\mathbf{2 5 2}$ \\
BF227507 & procollagen, type 1, alpha 2 & $\mathbf{5 6 3}$ & $\mathbf{2 0 3}$ \\
BB250811 & procollagen C-proteinase & $\mathbf{5 0 8}$ & $\mathbf{2 4 8}$ \\
& enhancer protein & & \\
NM_009378.1 & thrombomodulin & $\mathbf{5 0 7}$ & $\mathbf{2 4 7}$ \\
L42114.1 & growth differentiation factor 10 & $\mathbf{5 6 5}$ & $\mathbf{2 7 9}$ \\
AK011784.1 & insulin-like growth factor & $\mathbf{1 1 5 8}$ & $\mathbf{5 4 4}$ \\
BB787243 & inding protein 2 & & \\
& insulin-like growth factor & $\mathbf{5 8 0}$ & $\mathbf{2 3 6}$ \\
\hline
\end{tabular}

expression of procollagen type I, alpha 2, a gene in Table 2, did not significantly differ between the STR and CTL groups (data not shown). As a result, seven genes from Table 2 and Igf2 were decreased in the amygdala of CIS-induced depressed mice, and thus, we identified a total of eight genes as responders to CIS in the mouse amygdala. The microarray results obtained from ICR mice were confirmed in C57BL/6 mice with Q-PCR, indicating that the CIS-induced gene expression change in the amygdala did not differ between the two mouse strains.

In our laboratory, CIS-induced depressed mice displayed a somewhat wide range of behavior, although the mean value of immobility of the STR group was statistically different from the CTL group. For example, a few mice showed a relatively lower duration of immobility, which was comparable to the duration of the higher immobility CTL group. We used to exclude these lower duration mice from the CIS-induced depressed group for further experimentation. In the present study, for histological examination, we prepared new model animals according to the scheme depicted in Figure 1A and confirmed significant mean differences both in sucrose preference and immobility duration between the CTL and STR groups (Table 3). Then we selected six STR mice from two end points of the immobility spectrum and three mice from the CTL group to see whether CISresponsive gene expression was different according to the behavioral observations. Three mice showing the highest immobility duration from the STR group were categorized as STR-higher immobility (HI) mice, and three mice showing the lowest immobility duration from the STR group were considered STR-lower immobility (LI) mice. CTL-mean immobility (MI) mice consisted of mice with immobility duration in the mean range of the CTL group. From immunohistochemistry (IHC), we found that the overall expression level of osteoglycin was decreased in the amygdala of the STR-HI mice,

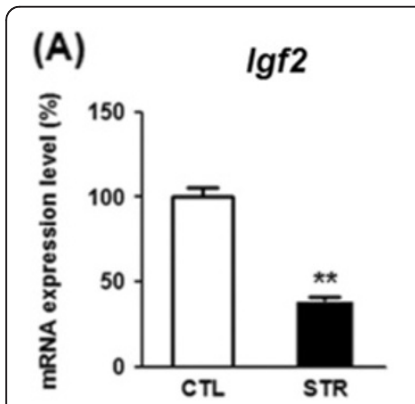

(E)

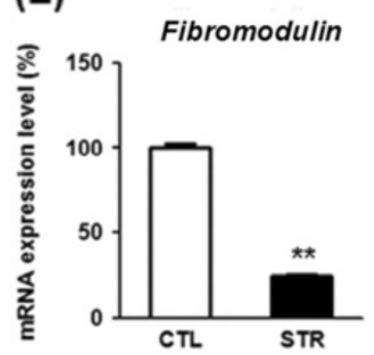

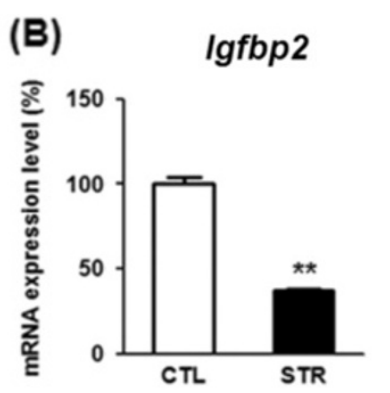

(F)

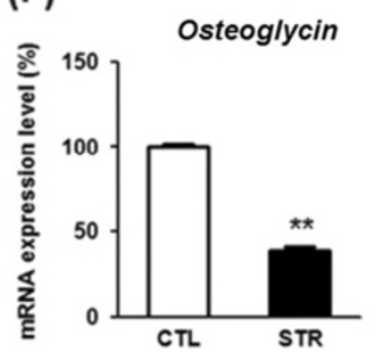

(C)

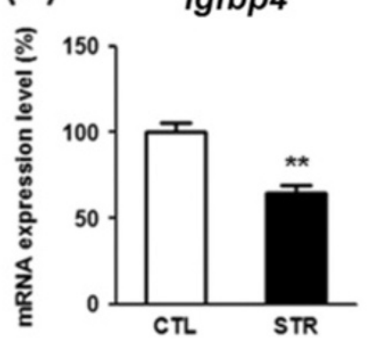

(G)

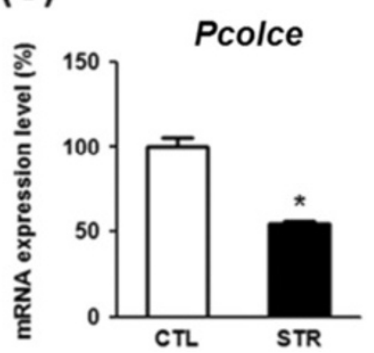

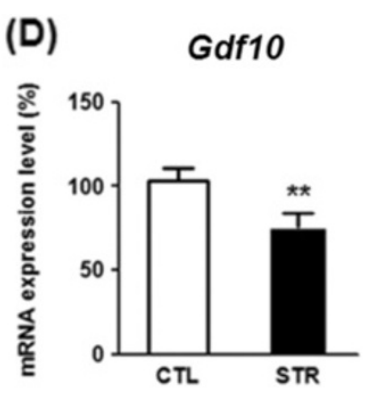

(H)

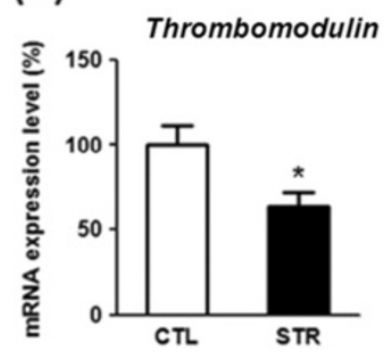

Figure 2 The results of Q-PCR analysis of selected genes using total RNA from the amygdala from stressed (STR, $n=9)$ and control $(\mathbf{C T L}, \mathbf{n}=9$ ) mice. Insulin-like growth factor 2 (Igf2) (A), Insulin-like growth factor binding protein-2 (Igfbp2) (B), Igfbp4 (C), growth differentiation factor $10($ Gdf10) (D), fibromodulin (E), osteoglycin (F), procollagen C-proteinase enhancer protein (Pcolce) (G), and thrombomodulin (H) mRNAs were significantly decreased in the amygdala of STR mice. Data are the means \pm SEM. ${ }^{*}, \mathrm{P}<0.05 ;{ }^{* *}, \mathrm{P}<0.01$ between the CTL and STR groups. 
Table 3 The effect of chronic immobilization stress on mouse depression-like behaviors. (Results are expressed as mean $\pm \mathrm{SEM}$ )

\begin{tabular}{llll}
\hline Behavioral Parameters & Control & Stress & $\boldsymbol{P}$ value \\
\hline & $(\mathbf{n = 1 0 )}$ & $\mathbf{( n = 1 5 )}$ & \\
\hline Sucrose Preference (\%) & $104.7 \pm 4.9$ & $79.4 \pm 5.8$ & $<0.001$ \\
(Sucrose Preference Test) & & & \\
Range & $80-130$ & $54-105$ & \\
Immobility Duration (s) & $137 \pm 5.3$ & $178.6 \pm 6.5$ & $<0.001$ \\
(Forced Swim Test) & & & \\
Range & $111-159$ & $125-209$ & \\
\hline
\end{tabular}

specifically in the central, basal, and medial parts compared with the CTL-MI mice (Figure 3). However, there was no significant difference between the CTL-MI and STR-LI mice. The expression of fibromodulin was significantly decreased in these three parts of the amygdala in STR-HI mice but not in the STR-LI mice, and this pattern was very similar to that of osteoglycin (Figure 4). Protein expression of Igf2 and Igfbp2 appeared to be specific to the central nucleus in the amygdala, and expression was also decreased only in the STR-HI mice (Figure 5).
To observe the behavioral differences in mice used for IHC, we reanalyzed these data as shown in Figure 6. STR-HI mice showed a significant difference in immobility duration compared to CTL-MI and STR-LI mice, and the mean value of STR-LI mice was similar to that of CTL-MI mice (Figure 6B), because this selection was done using their immobility duration data. The mean value of sucrose preference (Figure 6A) in STR-LI mice was slightly higher than STR-HI mice and lower than CTL-MI mice but the difference was not significant, indicating that mice undergoing CIS were different from those in the CTL group. However, STR-HI mice showed a significantly lower sucrose preference compared with CTL-MI mice (Figure 6A).

Statistically significant decreases in protein expression of four candidates were only found in the amygdala of STR-HI mice but not in STR-LI mice. These protein expression patterns suggest that the decreases in the amygdala may be closely correlated with the degree of behavioral changes caused by CIS.

\section{Discussion}

Using cDNA microarray and Q-PCR analysis, we identified eight genes that were decreased in the amygdala of

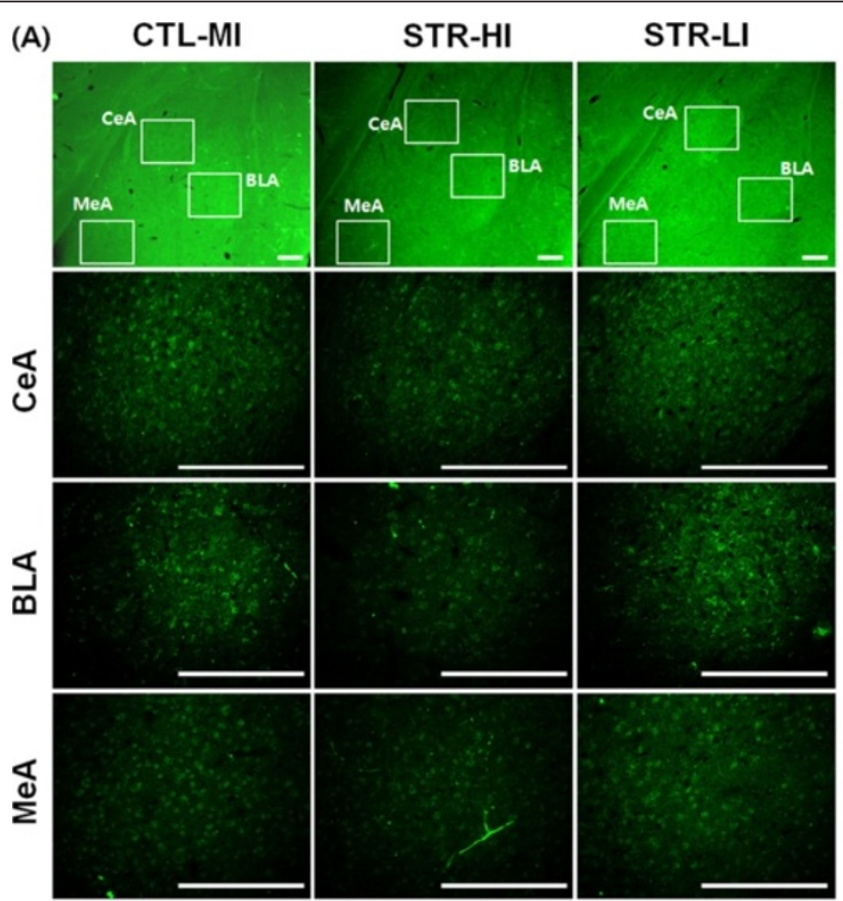

(B)
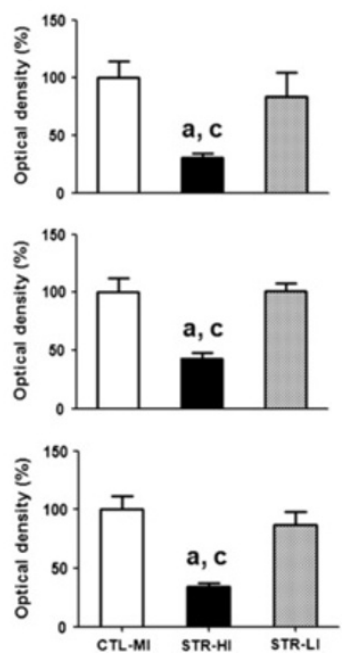

Figure 3 The expression of osteoglycin in the amygdala was analyzed using immunohistochemistry (IHC) in the three groups; CTL-MI STR-HI, and STR-LI. The representative IHC result shows specific decreased expression of osteoglycin in the central (CeA), basolateral (BLA), and medial (MeA) nuclei of the amygdala of STR-HI mice (A). The fluorescence signal density of each nucleus was quantified using the Image $J$ program $(\mathrm{NIH}),(B)$ and the strength of $\mathrm{IHC}$ was evaluated with one-way ANOVA and Tukey's multiple-comparison test among groups. All tested nuclei showed significant differences in signal intensity among groups ( $F$ values of CeA, BLA, and MeA were 8.79, 14.70, and 13.55, respectively). As a result, only STR-HI mice showed significant signal decreases in each nucleus. Data are the means \pm SEM $(n=3)$. a, $P<0.05$ between CTL-MI and STR-HI mice; C, P $<0.05$ between STR-HI and STR-LI mice. Scale bars are $200 \mu \mathrm{m}$. 


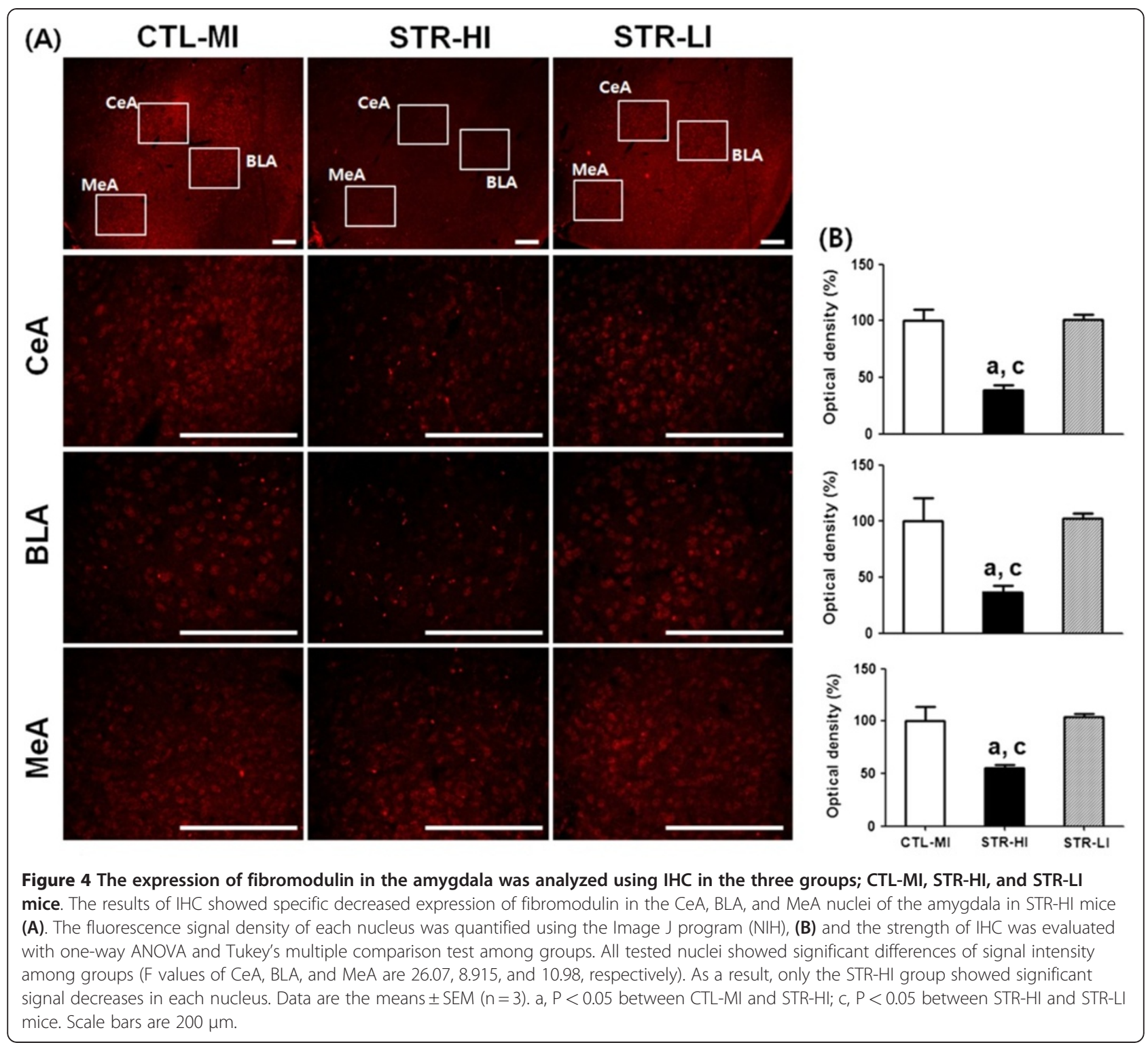

CIS-induced depressed mice. Four genes were further characterized using an immunohistological approach, which showed that protein expression was specifically reduced in the amygdala of STR-HI mice but not in STR-LI mice, even though both groups experienced the same CIS protocol.

Almost all organisms feel threats of homeostatic imbalance, and intrinsic stress responses begin to work during threats to cope with the stressors [20]. The stress response starts when the brain receives information about stressors through sensory mechanisms scattered throughout the body and activates specific pathways that were imprinted early in physiological development. In this way, organisms can maintain homeostatic conditions in stressful environments [6]. However, repetitive, longlasting stress can evoke depressive behaviors directed by the brain [21]. The brain has structural and functional plasticity that can result in both adaptive and maladaptive consequences [22]. It is now widely accepted that intense emotional events or chronic exposure to stressful experiences can create traumatic memories and even result in the development of mood and anxiety disorders, including post-traumatic stress disorder (PTSD) and major depressive disorder [22-24]. Depression has been reported to be the disease for which drugs are most frequently prescribed and is notorious for leading to many disabilities [25].

Research groups that consider stress as the main cause of depression have attempted to elucidate how stress results in structural and functional changes in specific brain regions and to develop innovative prevention and therapeutic strategies based on their findings [26]. 


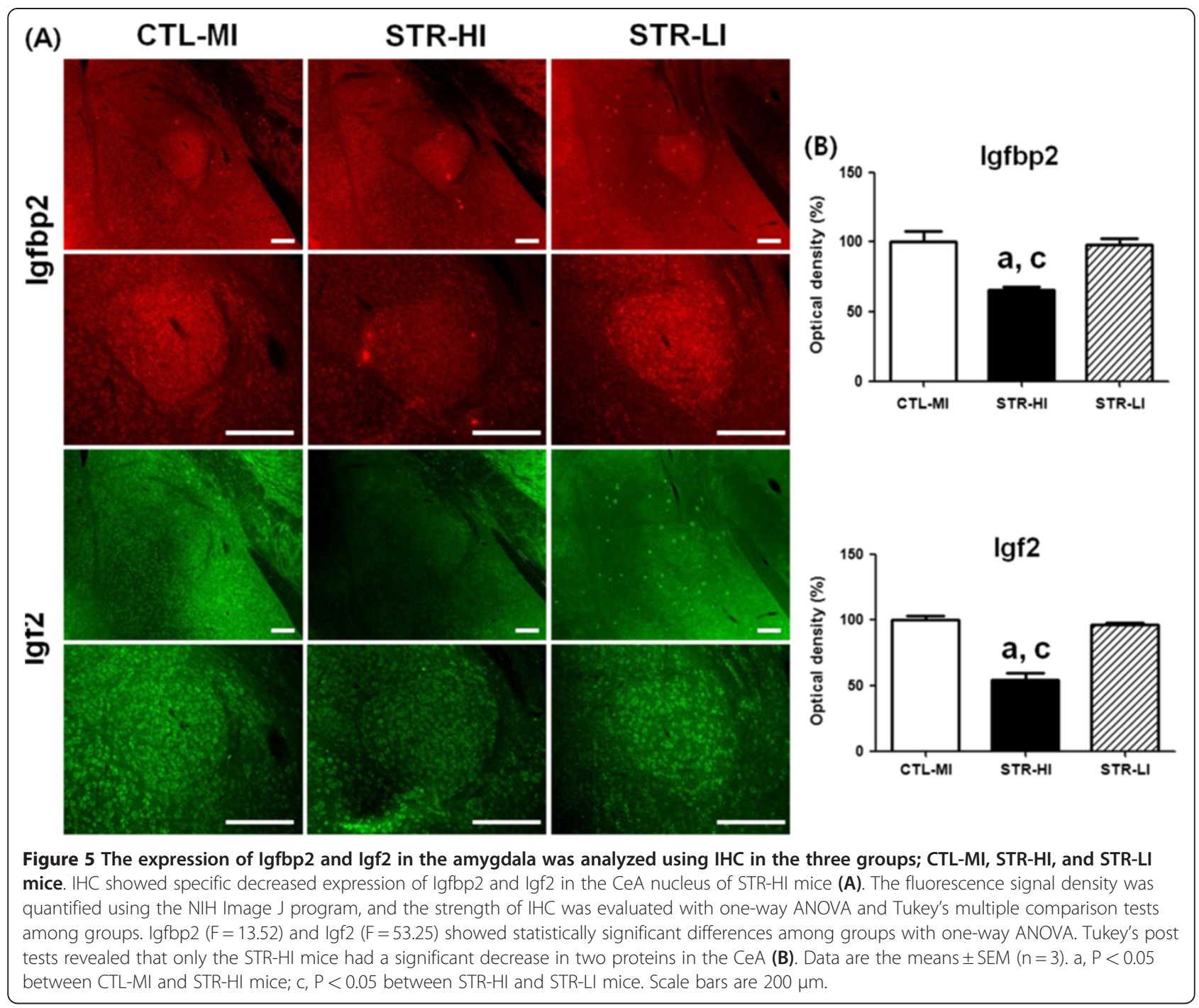

Recently, large-scale gene expression analysis has been performed using animal models of endogenous depression and chronic stress to test the monoamine hypothesis and find a common pathological mechanism [5]. However, the authors found no significant difference in the expression of monoaminergic transmission-related genes in either model, and very few overlapping, expression-changing genes in the two models, implicating divergent mechanisms between endogenous depression and stress-induced behavioral changes [5]. Although the authors failed to find a common pathological event, a few genes identified in that study as stress responsive were also found in our studies. For example, Igf2 was identified in this study (Figure 2), and transthyretin was previously identified [10]. Thus, these genes could be classified into chronic stress-responsive genes, and we may be able to obtain more plausible information about how stress evokes depression by studying the roles of such genes.
We carried out a microarray assay using an Affymetrix DNA chip, the GeneChip ${ }^{\circledR}$ Mouse Expression Set 430 2.0, which has almost 40,000 probes. However, as shown in Table 2, only 10 probes were selected as CIS responders, with the criteria that expression was relatively high and more than 2-fold different between the CTL and STR groups. All probes listed in Table 2 decreased in the stressed amygdala and were categorized as ECM proteins or trophic factors by their known functions. This result was very impressive and interesting, and the nature of the amygdala suggests a possible reason. The amygdala mainly consists of four nuclei that cross-talk among each other and have neural connections with other brain areas [6]. The prefrontal cortex plays a major role in recognizing environments and making decisions, but the amygdala is a key player that stores and consolidates emotional memory and behavioral patterns [24]. Almost all external stimuli afferent to the cerebral cortex affect the amygdala complex before being processed in 

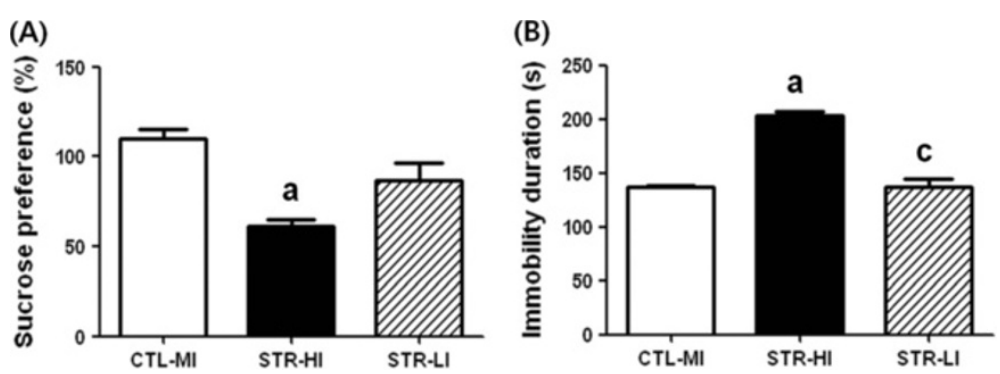

Figure 6 The sucrose preference and immobility duration of the mice selected for immunohistochemical analyses. Statistical significance was evaluated with one-way ANOVA and Tukey's post-hoc test. There were significant differences in sucrose preference $(\mathbf{A}, F=13.30, P=0.0062)$ and immobility duration ( $\mathbf{B}, F=52.59, P=0.0002)$ among the groups. From Tukey's post-hoc tests, STR-HI mice showed a statistically significant difference compared to the CTL-MI mice in the two parameters but STR-LI mice did not show a significant difference from CTL-MI mice. Data are the means \pm SEM ( $n=3$ per group). a, $P<0.05$ between CTL-MI and STR-HI mice; $c, P<0.05$ between STR-HI and STR-LI mice.

the cerebral cortex [6]. Therefore, the amygdala has numerous neural circuits with various brain regions and plays an important role in the process of emotional performance and memory formation [27]. In addition, ECM proteins in the brain not only have supportive and trophic functions, but modulate cell functionality and plasticity as well as participate in brain architecture [28]. Proteoglycans and collagens are important components of the brain ECM that have multiple functions, including cell adhesion, neurite outgrowth, ECM assembly, and tumor cell invasion [29]. Fibromodulin and osteoglycin are small, leucine-rich proteoglycans [30-32]. Moreover, trophic factors are generally regarded as major components that impact neural plasticity [33]. Thus, decreased expression of such genes in the amygdala may affect amygdaloid neural plasticity, including neuronal morphological changes and afferent and/or efferent neural circuits participating in stress-related emotional behaviors, which may result in psychiatric illness.

Several lines of evidence support the concept that stressinduced functional and morphological alterations in the amygdala can evoke psychiatric illness, including anxiety disorder, depressive disorder, and PTSD [22,23,34-38]. Neurons in the medial amygdala (MeA) have decreased numbers of dendritic spines after stress exposure, but neurons in the basolateral amygdala (BLA) have increased numbers [35]. The dendritic hypertrophy of the BLA induced by stress is considered a quantifiable marker of neuronal structural pathology in bipolar disorder [36]. The decrease in dendritic spines in the MeA may be a maladaptive phenotype in stress-induced psychiatric patients, including non-regulated energy homeostasis and sensorimotor gating because the MeA has connections with the hypothalamus and basal ganglia $[20,39]$. In addition to the morphological alterations in the BLA after stress exposure, chronic stress causes neuronal hyperexcitability that facilitates fear learning $[37,38]$. In particular, the BLA has been examined because it has numerous executive connections with other brain regions including the prefrontal cortex, hippocampus, caudate nucleus, nucleus accumbens, and other amygdaloid nuclei $[24,40]$. Such changes occurring in the amygdala following stress exposure are different compared with changes in other brain regions. The most important difference is the duration of change. In other words, the morphological changes in the amygdala can persist for quite a long period after cessation of stressful situations, but morphological changes in the hippocampus and medial prefrontal cortex are reversible over a short period of time [24]. Furthermore, the persistent morphological changes in the BLA can be induced by an acute corticosterone treatment [34]. Changes in the amygdala frequently occur in concert with functional changes in other brain regions $[24,40]$. In addition, the responsiveness of each nucleus in the amygdala is heterogeneous [35]. In the present study, the common nucleus showing altered protein expression was the central amygdala (CeA) (Figures 4, 5 and 6), which has been investigated to a lesser extent than the MeA or BLA. However, the $\mathrm{CeA}$ is responsible for directing the behavioral, autonomic, and endocrine responses in an anxious state $[41,42]$. Therefore, specific roles of newly identified genes in relation to neural plasticity of the CeA during the stress response deserve further study, and it will also be necessary to investigate which regions will show changes in functional connections with the CeA.

A single situation may not result in the same response in different individuals, and this is true in both humans and animals. Some people are susceptible to PTSD, whereas others are not, even though they experienced the same severe disaster [22,23]. We occasionally found such a phenomenon in experimental animals that were subjected to CIS. To produce the CIS-induced depressed animal model, we purchased inbred mice of the same age and treated them with identical conditions. Moreover, behavioral assessments of all mice after the CIS regimen were simultaneously conducted with the same protocol. Although mice experienced the same CIS 
regimen, their behavioral consequence had a somewhat wider range compared with the CTL mice (Table 3). In this study, we selected mice for immunohistological analyses of four candidates from the STR and CTL groups using their immobility duration and found that protein expression of candidates was significantly decreased in the STR-HI mice but not in the STR-LI mice (Figures 3, 4 and 5). Because immobility in the FST is considered a representative depression behavior [43], the difference in the immobility duration in the STR group can be interpreted as individual stress sensitivity. The mice used in this study had the same genetic background. Thus, individual stress sensitivity may be an acquired trait, and we hypothesize that individual stress vulnerability is closely correlated with the stress sensitivity of one's amygdala. Furthermore, the four genes reported in our current study may, at least in part, play a role in emotional behavioral changes related to stress vulnerability.

\section{Conclusions}

In summary, we identified eight genes as stress responders in the amygdala using a CIS-induced depression model and confirmed that decreased protein expression of the products of four genes was found in the STR-HI mice. Thus, these four genes may be involved in the chronic stress-induced depressive behaviors and acquired stress vulnerability of the amygdala. Therefore, further study is necessary to investigate the neural connections that originate in the CeA where gene expression levels decreased, because communication between the amygdala and other brain regions is indispensible for the exact function of the amygdala. Moreover, evaluation of the effect of gene expression changes on neural plasticity can help us understand the meaning of gene expression changes in the amygdala of CIS-induced depressed mice.

\begin{abstract}
Abbreviations
BLA: Basolateral nucleus of the amygdala; CeA: Central nucleus of the amygdala; CIS: Chronic immobilization stress; CTL: Control group; CTLMl: Control-mean immobility; DC: Decapitation; ECM: Extracellular matrix; FST: Forced swim test; Gdf10: Growth differentiation factor 10; Igf2: Insulinlike growth factor II; Igfbp2: Insulin-like growth factor binding protein 2; IHC: Immunohistochemistry; MeA: Medial nucleus of the amygdala; Pcolce: Procollagen C-proteinase enhancer protein; PTSD: Post-traumatic stress disorder; Q-PCR: Quantitative PCR; SPT: Sucrose preference test; STR: Stressed group; STR-HI: Stressed-higher immobility; STR-LI: Stressedlower immobility.
\end{abstract}

\section{Acknowledgements}

This research was supported by the Basic Science Research Program through the National Research Foundation of Korea (NRF) funded by the Ministry of Education, Science and Technology (2011-0003358).

\section{Authors' contributions}

SJ carried out CIS, behavioral assessments, and made the outline of the draft. $Y L$ set up the protocol for SPT and participated in behavioral assessments. GK created a protocol for $\mathrm{IHC}$ and participated in the $\mathrm{IHC}$ process. HS participated in the CIS procedure and assisted in animal care. DHL carried out statistical analyses. GSR participated in the IHC procedure and helped draft the manuscript. SSK participated in the design of the study and analysis of microarray data. GJC participated in the process of $\mathrm{HHC}$ and analysis of the results. WSC participated in the design of the study and helped draft the manuscript. HJK designed the study and wrote the manuscript. All authors read and approved the final manuscript.

Received: 29 February 2012 Accepted: 6 June 2012

Published: 6 June 2012

References

1. Boudarene M, Legros JJ, Timsit-Berthier M: Study of the stress response: role of anxiety, cortisol and DHEAs. Encephale 2002, 28(2):139-146.

2. Northoff G, Wiebking C, Feinberg T, Panksepp J: The 'resting-state hypothesis' of major depressive disorder-A translational subcorticalcortical framework for a system disorder. Neurosci Biobehav Rev 2011, 35(9):1929-1945.

3. Zychlinska M, Florkowski A, Furmanska A, Dudak M, Gajda A: Influence of therapy effectiveness of neurotic and stress disorders on selected aspects of psychological function. Pol Merkur Lekarski 2008, 25(Suppl 1):20-22.

4. Parent MA, Wang L, Su J, Netoff T, Yuan LL: Identification of the hippocampal input to medial prefrontal cortex in vitro. Cereb Cortex 2010, 20(2):393-403.

5. Andrus BM, Blizinsky K, Vedell PT, Dennis K, Shukla PK, Schaffer DJ, Radulovic J, Churchill GA, Redei EE: Gene expression patterns in the hippocampus and amygdala of endogenous depression and chronic stress models. Mol Psychiatry 2012, 17(1):49-61.

6. Rodrigues SM, LeDoux JE, Sapolsky RM: The influence of stress hormones on fear circuitry. Annu Rev Neurosci 2009, 32:289-313.

7. Amunts K, Kedo O, Kindler M, Pieperhoff P, Mohlberg H, Shah NJ, Habel U, Schneider F, Zilles K: Cytoarchitectonic mapping of the human amygdala, hippocampal region and entorhinal cortex: intersubject variability and probability maps. Anat Embryol (Berl) 2005, 210(5-6):343-352.

8. Tye KM, Prakash R, Kim SY, Fenno LE, Grosenick L, Zarabi H, Thompson KR, Gradinaru V, Ramakrishnan C, Deisseroth K: Amygdala circuitry mediating reversible and bidirectional control of anxiety. Nature 2011, 471(7338):358-362.

9. Sibille E, Wang Y, Joeyen-Waldorf J, Gaiteri C, Surget A, Oh S, Belzung C, Tseng GC, Lewis DA: A molecular signature of depression in the amygdala. Am J Psychiatry 2009, 166(9):1011-1024.

10. Joo Y, Choi KM, Lee YH, Kim G, Lee DH, Roh GS, Kang SS, Cho GJ, Choi WS, Kim HJ: Chronic immobilization stress induces anxiety- and depressionlike behaviors and decreases transthyretin in the mouse cortex. Neurosci Lett 2009, 461(2):121-125.

11. Jo M, Gieske MC, Payne CE, Wheeler-Price SE, Gieske JB, Ignatius IV, Curry TE $\mathrm{Jr}, \mathrm{Ko} \mathrm{C}$ : Development and application of a rat ovarian gene expression database. Endocrinology 2004, 145(11):5384-5396.

12. Sakata $K$, Jin $L$, Jha S: Lack of promoter IV-driven BDNF transcription results in depression-like behavior. Genes Brain Behav 2010, 9(7):712-721.

13. Paxinos G, Franklin K: The mouse brain in stereotaxic coordinates. San Diego: Academic; 2001

14. Kim G, Lee Y, Jeong EY, Jung S, Son H, Lee DH, Roh GS, Kang SS, Cho GJ, Choi WS, et al: Acute stress responsive RGS proteins in the mouse brain. Mol Cells 2010, 30(2):161-165.

15. Wurtz T, Houari S, Mauro N, MacDougall M, Peters H, Berdal A: Fluoride at non-toxic dose affects odontoblast gene expression in vitro. Toxicology 2008, 249(1):26-34.

16. Hamajima S, Hiratsuka K, Kiyama-Kishikawa M, Tagawa T, Kawahara M, Ohta M, Sasahara H, Abiko Y: Effect of low-level laser irradiation on osteoglycin gene expression in osteoblasts. Lasers Med Sci 2003, 18(2):78-82.

17. Song D, Ye X, Xu H, Liu SF: Activation of endothelial intrinsic NF-\{kappa\}B pathway impairs protein $C$ anticoagulation mechanism and promotes coagulation in endotoxemic mice. Blood 2009, 114(12):2521-2529.

18. Boutinaud M, Shand JH, Park MA, Phillips K, Beattie J, Flint DJ, Allan GJ: A quantitative RT-PCR study of the mRNA expression profile of the IGF axis during mammary gland development. J Mol Endocrinol 2004, 33(1):195-207.

19. Jeon BT, Shin HJ, Kim JB, Kim YK, Lee DH, Kim KH, Kim HJ, Kang SS, Cho GJ, Choi WS, et al: Adiponectin protects hippocampal neurons against kainic acid-induced excitotoxicity. Brain Res Rev 2009, 61(2):81-88.

20. Solomon MB, Jones K, Packard BA, Herman JP: The medial amygdala modulates body weight but not neuroendocrine responses to chronic stress. J Neuroendocrinol 2010, 22(1):13-23. 
21. Wood GE, Norris EH, Waters E, Stoldt JT, McEwen BS: Chronic immobilization stress alters aspects of emotionality and associative learning in the rat. Behav Neurosci 2008, 122(2):282-292.

22. Mitra R, Adamec R, Sapolsky R: Resilience against predator stress and dendritic morphology of amygdala neurons. Behav Brain Res 2009, 205(2):535-543.

23. Admon R, Lubin G, Stern O, Rosenberg K, Sela L, Ben-Ami H, Hendler T: Human vulnerability to stress depends on amygdala's predisposition and hippocampal plasticity. Proc Natl Acad Sci U S A 2009, 106(33):14120-14125.

24. Roozendaal B, McEwen BS, Chattarji S: Stress, memory and the amygdala. Nat Rev Neurosci 2009, 10(6):423-433.

25. Phillips MR, Yang G, Zhang Y, Wang L, Ji H, Zhou M: Risk factors for suicide in China: a national case-control psychological autopsy study. Lancet 2002, 360(9347):1728-1736.

26. aan het Rot M, Mathew SJ, Charney DS: Neurobiological mechanisms in major depressive disorder. CMAJ 2009, 180(3):305-313.

27. LeDoux J: The emotional brain, fear, and the amygdala. Cell Mol Neurobiol 2003, 23(4-5):727-738

28. Bonneh-Barkay D, Wiley CA: Brain extracellular matrix in neurodegeneration. Brain Pathol 2009, 19(4):573-585.

29. Ruoslahti E: Brain extracellular matrix. Glycobiology 1996, 6(5):489-492.

30. lozzo RV: The biology of the small leucine-rich proteoglycans. Functional network of interactive proteins. J Biol Chem 1999, 274(27):18843-18846.

31. Font B, Eichenberger D, Goldschmidt D, Boutillon MM, Hulmes DJ: Structural requirements for fibromodulin binding to collagen and the control of type I collagen fibrillogenesis-critical roles for disulphide bonding and the C-terminal region. Eur J Biochem 1998, 254(3):580-587.

32. Kukita A, Bonewald L, Rosen D, Seyedin S, Mundy GR, Roodman GD: Osteoinductive factor inhibits formation of human osteoclast-like cells. Proc Natl Acad Sci U S A 1990, 87(8):3023-3026.

33. Russo VC, Gluckman PD, Feldman EL, Werther GA: The insulin-like growth factor system and its pleiotropic functions in brain. Endocr Rev 2005, 26(7):916-943

34. Mitra R, Sapolsky RM: Acute corticosterone treatment is sufficient to induce anxiety and amygdaloid dendritic hypertrophy. Proc Natl Acad Sci U S A 2008, 105(14):5573-5578.

35. Bennur S, Rao Shankaranarayana BS, Pawlak R, Strickland S, McEwen BS, Chattarji S: Stress-induced spine loss in the medial amygdala is mediated by tissue-plasminogen activator. Neuroscience 2007, 144(1):8-16.

36. Johnson SA, Wang JF, Sun X, McEwen BS, Chattarji S, Young LT: Lithium treatment prevents stress-induced dendritic remodeling in the rodent amygdala. Neuroscience 2009, 163(1):34-39.

37. Rodriguez Manzanares PA, Isoardi NA, Carrer HF, Molina VA: Previous stress facilitates fear memory, attenuates GABAergic inhibition, and increases synaptic plasticity in the rat basolateral amygdala. J Neurosci 2005 25(38):8725-8734.

38. Rosenkranz JA, Venheim ER, Padival M: Chronic stress causes amygdala hyperexcitability in rodents. Biol Psychiatry 2010, 67(12):1128-1136.

39. Vinkers CH, Bijlsma EY, Houtepen LC, Westphal KG, Veening JG, Groenink L, Olivier B: Medial amygdala lesions differentially influence stress responsivity and sensorimotor gating in rats. Physiol Behav 2010 99(3):395-401.

40. Shansky RM, Hamo C, Hof PR, McEwen BS, Morrison JH: Stress-induced dendritic remodeling in the prefrontal cortex is circuit specific. Cereb Cortex 2009, 19(10):2479-2484.

41. Bishop SJ: Neurocognitive mechanisms of anxiety: an integrative account. Trends Cogn Sci 2007, 11(7):307-316.

42. Millan MJ: The neurobiology and control of anxious states. Prog Neurobiol 2003, 70(2):83-244

43. Perona MT, Waters S, Hall FS, Sora I, Lesch KP, Murphy DL, Caron M, Uhl GR: Animal models of depression in dopamine, serotonin, and norepinephrine transporter knockout mice: prominent effects of dopamine transporter deletions. Behav Pharmacol 2008, 19(5-6):566-574.

doi:10.1186/1471-2202-13-58

Cite this article as: Jung et al:: Decreased expression of extracellular matrix proteins and trophic factors in the amygdala complex of depressed mice after chronic immobilization stress. BMC Neuroscience 2012 13:58

\section{Submit your next manuscript to BioMed Central and take full advantage of:}

- Convenient online submission

- Thorough peer review

- No space constraints or color figure charges

- Immediate publication on acceptance

- Inclusion in PubMed, CAS, Scopus and Google Scholar

- Research which is freely available for redistribution 